\title{
On finite permutation groups with the same orbits on unordered sets
}

\author{
By \\ JoHANNES SIEMONS and ASCHER WAGNER
}

1. Introduction. A permutation group $G$ acting on a set $\Omega$ induces a permutation group on the unordered sets of $k$ distinct points. If $H$ is another permutation group on $\Omega$ we shall write $H \stackrel{k}{\approx} G$ if $H$ and $G$ have the same orbits on the unordered sets of $k$ points. Bercov and Hobby [2] have shown that for infinite groups $H \stackrel{k}{\approx} G$ implies $H \stackrel{l}{\approx} G$ if $l<k$. In [9] we have shown that this result is also true for finite groups, with the obviously necessary condition that $k \leqq \frac{1}{2}|\Omega|$. In [9] it is also shown that for finite groups $H \stackrel{2}{\approx} G$ implies that $H$ and $G$ are either both primitive or both imprimitive with the same blocks of imprimitivity.

If $H$ and $G$ have the same orbits on all subsets of $\Omega$ we shall say that $H$ and $G$ are orbit equivalent and write $H \approx G$. Orbit equivalence for groups acting on quite arbitrary $\Omega$ has been considered by Betten [3]; the main results concern intransitive groups. In this paper we shall be concerned only with orbit equivalence for finite groups. In this case, of course, $H \approx G$ if, and only if, $H \stackrel{k}{\approx} G$ for all $k$.

Suppose that $H \approx G$ and that $L$ is a permutation group on a set $\Delta$. Then the direct products $H \times L$ and $G \times L$, acting naturally on $\Omega \cup \Delta$, are orbit equivalent and intransitive. Also, if $L$ is transitive on $\Delta$ the wreath products $H \chi L$ and $G \chi L$, acting naturally on the direct sum of $|\Delta|$ copies of $\Omega$, are orbit equivalent and imprimitive. This suggests that the basic situation to investigate is when $G$, hence also $H$, is primitive on $\Omega$.

Without loss of generality we may assume that $H \subset G$ since $H \approx G$ implies $H \approx\langle H, G\rangle$. Our main result is the following theorem.

Theorem A. Let $K$ be a finite primitive permutation group on a set $\Omega$. Let $H \subset K$ and $H \approx K$. Suppose there exists a prime $r$ dividing the order of $K$ but not the order of $H$. Then only the following possibilities exist:

\begin{tabular}{lllllccc}
\hline & $H$ & $K$ & $|\Omega|$ & $r$ & 2-sets & 3-sets & 4-sets \\
\hline (i) & $\mathbb{A}_{3}$ & $\mathbb{S}_{3}$ & 3 & 2 & & & \\
(ii) & $C_{5}$ & $D_{10}$ & 5 & 2 & $5 ; 5$ & & \\
(iii) & $A_{1}(5)$ & $\mathbb{S}_{5}$ & 5 & 3 & 10 & & \\
(iv) & $A_{1}(8)$ & $\Gamma A_{1}(8)$ & 8 & 3 & 28 & 56 & $14 ; 56$ \\
(v) & $\boldsymbol{A}_{1}(8)$ & $2^{3} \backslash P S L_{3}(2)$ & 8 & 3 & 28 & 56 & $14 ; 56$ \\
(vi) & $P G L_{2}(8)$ & $\mathbb{A}_{9}$ or $\mathbb{S}_{9}$ & 9 & 5 & 36 & 84 & 126 \\
(vii) & $P \Gamma L_{2}(8)$ & $\mathbb{A}_{9}$ or $\mathbb{S}_{9}$ & 9 & 5 & 36 & 84 & 126 \\
\hline
\end{tabular}


The notation is explained in Section 2. The last three columns of the table give the orbit lengths. The proof of Theorem A is elementary.

In Section 6 we prove a rather stronger version of Theorem A. This, however, makes use of the classification of 3-transitive groups.

Henceforth in this paper the symbols $K, H$ and $r$ shall always have the meaning given to them in Theorem A. Also $n$ shall denote $|\Omega|$.

Since all orbits of $H$ on subsets of $\Omega$ have length prime to $r$ it follows that given any $\Delta \subset \Omega$ there exists a Sylow $r$-subgroup of $K$ leaving $\Delta$ fixed as a set. This property is implicitly used throughout the proof.

2. Notation and results needed later. We consider a finite set $\Omega$ consisting of elements called points. Upper case Greek letters shall denote subsets of $\Omega$ and lower case Greek letters shall denote points of $\Omega$. A set of $k$ distinct points of $\Omega$ shall be called a $k$-set. The collection of all $k$-sets in $\Omega$ shall be denoted by $\Omega^{\{k\}}$. $\mathbb{S}^{\Omega}$ and $\mathbb{A}^{\Omega}$ denote respectively the symmetric and the alternating group on $\Omega$.

If $G$ is a group of permutations on $\Omega$ we denote by $G_{[4]}$ the subgroup of $G$ fixing $\Delta$ pointwise and by $G_{(\Delta)}$ the subgroup of $G$ fixing $\Delta$ as a set. We shall also say that $G_{[\Delta]}$ stabilizes $\Delta$ and that $G_{(\Delta)}$ fixes $\Delta$. If $G$ is transitive on $\Omega^{\{k\}}$ we shall say that $G$ is $k$ homogeneous.

If $G$ is an intransitive group on $\Omega$ and $\Gamma$ is a union of orbits of $G$ then $G^{\Gamma}$ denotes the homomorphic image of $G$ obtained as a permutation group by restricting $G$ to $\Gamma$.

In this paper all groups are permutation groups. Most of the groups which occur have a natural representation, e.g. $M_{11}$ is the 4-transitive group on 11 points. When a group occurs we shall only mention its representation if it is other than the natural one.

$C_{n}$ and $D_{2 n}$ denote respectively the cyclic group of order $n$ and the dihedral group of order $2 n$. Their natural representation is considered to be the transitive one on $n$ points. $2^{3} \backslash P S L_{3}(2)$ denotes the split extension of $C_{2} \times C_{2} \times C_{2}$ by $P S L_{3}(2)$ and its natural representation for us is as a 3 -transitive group on 8 points. The one dimensional linear and semilinear affine groups over $F_{q}$ shall be denoted by $A_{1}(q)$ and $\Gamma A_{1}(q)$ respectively and their natural representation is on the affine line with $q$ points. The rest of the notation is quite standard.

We shall have frequent occasion to use the following elementary and old results. Proofs of the first two may be found for example in [7], and of the third, slightly differently formulated, in [5 p. 68]. The fourth is proved in a similar manner to the third.

Result 2.1. Let $C$ and $D$ be permutation groups on a set $\Omega$ and let $\gamma$ and $\delta$ be two points of $\Omega$ and $p$ a given prime. If $C$ and $D$ both have order a power of $p$ and have as their only fixed point $\gamma$ and $\delta$ respectively, then $\gamma$ and $\delta$ belong to the same orbit of $\langle C, D\rangle$.

Result 2.2. Let $G$ be a permutation group on a set $\Omega$ of $n$ points; let $p$ be a given prime and $k$ a given integer with $1 \leqq k<n$. If for every subset $\Gamma$ of $\Omega$ with $|\Gamma|=k$, there exists a subgroup $C$ of $G$ which has order a power of $p$ and which fixes the points of $\Gamma$ and no further points, then $G$ is $k$-transitive on $\Omega$. 
Result 2.3. Let $G$ be a permutation group on a set $\Omega$. Let $\Sigma$ be a subset of $\Omega$ and let $\Gamma=\left\{\gamma_{1}, \ldots, \gamma_{s}\right\}$ and $A=\left\{\delta_{1}, \ldots, \delta_{s}\right\}$ be subsets of $\Sigma$. Suppose there exists an $x$ in $G$ with $\gamma_{i}^{x}=\delta_{i}, i=1, \ldots$, s. If $\Sigma$ is the set of all points stabilized by a Sylow p-subgroup $S$ of $G_{[d]}$ then there exists a $y \in N_{G}(S) \subseteq G_{(\Sigma)}$ with $\gamma_{i}^{y}=\delta_{i}, i=1, \ldots, s$.

Result 2.4. Let $G$ be a permutation group on a set $\Omega$ and let $S$ be a Sylow p-subgroup of $G$. Let $\Gamma$ and $\Delta$ be unions of $S$-orbits and suppose there exists an $x$ in $G$ with $\Gamma^{x}=\Delta$. Then there exists a $y$ in $N_{G}(S)$ with $\Gamma^{y}=\Delta$.

3. The fixed points of Sylow r-subgroups. Let $G$ be a permutation group on $\Omega$ and let $\Sigma \subset \Omega, \sigma \in \Sigma$ and $\alpha \in \Omega$. We shall say that $\alpha$ is $\Sigma$-linked to $\sigma$ if there exists an element in $G$ stabilizing $\Sigma-\{\sigma\}$ and mapping $\alpha$ onto $\sigma$. In particular every point of $\Sigma$ is $\Sigma$-linked to itself.

Lemma 3.1. Let $S$ be a Sylow r-subgroup of $K$ and let $\Sigma$ be the set of points stabilized by $S$. Then $|\Sigma| \geqq r-1$ and every point of $\Omega-\Sigma$ is $\Sigma$-linked to at least $r-1$ points of $\Sigma$.

Proof. That $|\Sigma| \geqq r-1$ follows from the fact that a $r$-subgroup fixing a $(r-1)$-set stabilizes it.

Let $\alpha \in \Omega-\Sigma$ and let $\Delta=\Sigma \cup\{\alpha\}$. Let $S^{\prime}$ be a Sylow $r$-subgroup fixing $\Delta$. Suppose $S^{\prime}$ fixes $\alpha$. Then $S^{\prime}$ fixes $\Sigma$ and, since $S$ and $S^{\prime}$ have equally many fixed points, $S^{\prime}$ cannot be the identity on $\Sigma$. However $K_{(\Sigma)}^{\Sigma} \cong K_{(\Sigma)} / K_{[\Sigma]}$, hence the order of $K_{(\Sigma)}^{\Sigma}$ cannot contain $r$ as a factor. Consequently $S^{\prime}$ does not fix $\alpha$. Hence $S^{\prime}$ contains a permutation $x$ which permutes cyclically the ordered set $\left\{\alpha, \sigma_{1}, \sigma_{2}, \ldots, \sigma_{r-1}\right\}$ where $\sigma_{1}, \sigma_{2}, \ldots, \sigma_{r-1} \in \Sigma$. Then $S_{i}=x^{-i} S x^{i}$ has as its set of fixed points $\Sigma_{i}=\Lambda-\left\{\sigma_{i}\right\}, i=1,2, \ldots, r-1$. Now $\Sigma \cap \Sigma_{i}=\Delta-\left\{\alpha, \sigma_{i}\right\}$ and it follows from Result 2.1 that $\left\langle S, S_{i}\right\rangle$ maps $\alpha$ onto $\sigma_{i}$ i.e. $\alpha$ and $\sigma_{i}$ are $\Sigma$-linked.

Lemma 3.2. Let $S$ be a Sylow r-subgroup of $K$ and let $\Sigma$ be the set of points stabilized by S. Suppose some point $\alpha \in \Omega-\Sigma$ is $\Sigma$-linked to at least two points of $\Sigma$. Then

(i) $K_{(\Sigma)}^{\Sigma}$ contains a transposition.

(ii) If $K_{(\Sigma)}^{\Sigma}$ is primitive then $K_{(\Sigma)}^{\Sigma}=\mathbb{S}^{\Sigma}$.

(iii) If $K_{(\Sigma)}^{\Sigma}$ is imprimitive with minimal blocks of imprimitivity $\Pi_{1}, \ldots, \Pi_{t}$, then $K_{(\Sigma)}^{\Sigma}$ contains the direct product $\mathbb{S}^{\Pi_{1}} \times \cdots \times \mathbb{S}^{\Pi_{t}}$.

Proof. Suppose $\alpha$ is $\Sigma$-linked to $\sigma_{1}, \sigma_{2} \in \Sigma$. Let $\Delta=\Sigma-\left\{\sigma_{1}, \sigma_{2}\right\}$. From the definition of $\Sigma$-linked it follows easily that there exists an $x \in K$ which stabilizes $\Delta$ and maps $\sigma_{1}$ onto $\sigma_{2}$. It follows from Result 2.3 that there exists a $y \in K_{(\Sigma)}$ stabilizing $\Delta$ and mapping $\sigma_{1}$ onto $\sigma_{2}$. Consequently $y$ is a transposition on $\Sigma$, proving (i). By a well-known theorem (e.g. Wielandt [10, Theorem 13.3]) (ii) follows and (iii) is a corollary of (ii).

Lemma 3.3. A Sylow r-subgroup of $K$ fixes exactly $r-1$ points. Also $K$ is $(r-1)$-transitive on $\Omega$.

P r o of. In view of Result 2.2, the second assertion of the lemma follows immediately from the first assertion. We shall now prove the first assertion. 
Let $\Sigma=\left\{\sigma_{1}, \ldots, \sigma_{s}\right\}$ be the set of points fixed by the Sylow $r$-subgroup $S$ of $K$. We shall assume throughout that $s>r-1$ since otherwise there is nothing to prove. As was noted in the proof of Lemma 3.1 $K_{(\Sigma)}^{\Sigma}$ does not contain any elements of order $r$.

We must now distinguish between cases.

The ca s e $r>2$. By Result $2.3 K_{(\Sigma)}^{\Sigma}$ is transitive and in view of Lemmas 3.1 and 3.2 we reach a contradiction if $K_{(\Sigma)}^{\Sigma}$ is primitive or imprimitive and minimal blocks of imprimitivity have length $\geqq r$.

The blocks clearly have length at least $r-1$ hence they have length $r-1$ and it follows that each point of $\Omega-\Sigma$ is $\Sigma$-linked to exactly $r-1$ points of $\Sigma$ all in the same block. Denote the blocks by $\Pi_{1}, \ldots, \Pi_{t}$ and let $\Lambda_{i}$ be the set of all points of $\Omega$ which are $\Sigma$-linked to all points of $\Pi_{i}$. The $\Lambda_{i}, i=1, \ldots, t$, are a partition of $\Omega$.

We claim that if $x \in K_{[\Sigma-\{\sigma\}]}$, where $\sigma \in \Pi_{i}$, then $x$ maps $\Lambda_{i}$ onto itself. Suppose $x$ maps $\alpha$ onto $\beta$ where $\alpha \in \Lambda_{i}$ and $\beta \in \Lambda_{j} \neq \Lambda_{i}$. Let $y$ be an element of $K_{[\Sigma-\{\sigma\}]}$ mapping $\sigma$ onto $\alpha$. Then $y x \in K_{[\Sigma-\{\sigma\}]}$ and maps $\sigma$ onto $\beta$. Consequently $\beta \in \Lambda_{i}$ which is a contradiction, establishing our claim.

Since $K$ is primitive on $\Omega$ there exists an $x \in K$ and an $\alpha, \beta \in \Lambda_{1}$ such that $\alpha^{x} \in \Lambda_{1}$ and $\beta^{x} \notin \Lambda_{1}$. Let $\sigma_{1}, \sigma_{2} \in \Pi_{1}$ and choose an $x_{1} \in K_{\left[\Sigma-\left\{\sigma_{1}\right\}\right]}$ such that $x_{1}$ maps $\alpha$ onto $\sigma_{1}$. Now choose an $x_{2} \in K_{\left[\Sigma-\left\{\sigma_{2}\right\}\right]}$ such that $x_{2}$ maps $\alpha^{x x_{1}}$ onto $\sigma_{2}$. Then $y=\left(x_{1} x_{2}\right)^{-1} x\left(x_{1} x_{2}\right)$ maps $\sigma_{1}$ onto $\sigma_{2}$. Let $\gamma=\beta^{x_{1} x_{2}}$ and $\delta=\beta^{x x_{1} x_{2}}$. Then $\gamma^{y}=\delta$. Also $\gamma \in \Lambda_{1}$ and $\delta \notin \Lambda_{1}$.

Suppose $r>3$. Let $\sigma_{3} \in \Pi_{1}$. Conjugating $y$ by a suitable element in $K_{\left[\Sigma-\left\{\sigma_{3}\right\}\right]}$ we obtain an element $z$ which maps $\sigma_{1}$ onto $\sigma_{2}$ and $\sigma_{3}$ onto an element outside $\Lambda_{1}$. Now conjugating $z$ by a suitable element in $K_{\left[\Sigma-\left\{\sigma_{j}\right\}\right]}$, where $\sigma_{j} \notin \Pi_{1}$, we obtain an element $w$ which maps $\sigma_{1}$ onto $\sigma_{2}$ and $\sigma_{3}$ onto $\sigma_{j}$. By Result 2.3 there exists an element in $K_{(\Sigma)}$ having the same action on $\sigma_{1}, \sigma_{2}, \sigma_{3}, \sigma_{j}$. This violates the imprimitivity of $K_{(\Sigma)}^{\Sigma}$.

Finally suppose $r=3$. Suppose $\lambda=\sigma_{2}^{y} \notin \Lambda_{1}$. Conjugating $y$ by a suitable element in $K_{\left[\Sigma-\left\{\sigma_{j}\right\}\right]}$, where $\sigma_{j} \notin \Pi_{1}$, we obtain an element $v: \sigma_{1} \rightarrow \sigma_{2} \rightarrow \sigma_{j}$. This leads to a contradiction as in the previous case. We now suppose $\lambda \in \Lambda_{1}$. Consider a Sylow $r$-subgroup $S^{\prime}$ fixing $\Sigma \cup\{\gamma\}$. From the argument used in the proofs of Lemmas 3.1 and 3.3 it is seen that there is an element $c$ of order 3 in $S^{\prime}$ permuting cyclically $\left\{\sigma_{1}, \sigma_{2}, \gamma\right\}$. We may suppose that $c: \sigma_{1} \rightarrow \sigma_{2} \rightarrow \gamma \rightarrow \sigma_{1}$. Then $u=y^{-1} c y: \delta \rightarrow \alpha_{2} \rightarrow \lambda \rightarrow \delta$. Conjugating $u$ by a suitable element in $K_{\left[\Sigma-\left\{\sigma_{1}\right\}\right]}$ we reach the situation considered at the beginning of this paragraph. This was seen to lead to a contradiction.

The cas e $r=2$. If some point of $\Omega-\Sigma$ is $\Sigma$-linked to two points of $\Sigma$, then $K_{(\Sigma)}^{\Sigma}$ contains a transposition contrary to our assumption. Consequently every point of $\Omega-\Sigma$ is $\Sigma$-linked to exactly one point of $\Sigma$. Let $A_{i}$ be the set of all points of $\Omega$ which are $\Sigma$-linked to $\sigma_{l}, i=1, \ldots, s$. As in the previous case the $\Lambda_{i}$ are a partition; also every element of $K_{\left[\Sigma-\left\{\sigma_{i}\right\}\right]}$ maps $\Lambda_{i}$ onto itself.

Let $N$ be the subgroup of $K$ generated by all involutions in $K$. Since $N \leqq K$ and $K$ is primitive on $\Omega, N$ is transitive on $\Omega$. Consequently some involution, say $y$, interchanges a point $\alpha \in \Lambda_{l}$ with a point $\beta \in \Lambda_{j} \neq \Lambda_{i}$.

Choose an $x_{i} \in K_{\left[\Sigma-\left\{\sigma_{k}\right\}\right]}$ such that $x_{i}$ maps $\alpha$ onto $\sigma_{i}$. Let $\beta^{x_{i}} \in \Lambda_{k}$. Then $\Lambda_{k} \neq \Lambda_{i}$. Choose an $x_{k} \in K_{\left[\Sigma-\left\{\sigma_{k}\right\}\right]}$ such that $x_{k}$ maps $\beta^{x_{i}}$ onto $\sigma_{k}$. Then $\left(x_{i} x_{k}\right)^{-1} y\left(x_{i} x_{k}\right)$ inter- 
changes $\sigma_{i}$ and $\sigma_{k}$. By Result 2.3 there exists an element in $K_{(\Sigma)}$ also interchanging $\sigma_{l}$ and $\sigma_{k}$. Consequently $K_{(\Sigma)}^{\Sigma}$ contains an element of order 2 , which is a contradiction.

This completes the proof of the lemma.

4. The high transitivity of $\boldsymbol{K}$. In the previous section we have shown that $K$ is $(r-1)$-fold transitive. Here we shall prove that $K$ is $(2 r-1)$-fold homogeneous in general. The number of $K$-orbits on $\Omega^{\{i\}}$ shall be denoted by $n_{i}(K)$.

Proposition 4.1. If $K$ is a group as defined in section 1, then i) $n_{r}(K)=n_{2 r-1}(K)$ and ii): If $4 r-2 \leqq n$, then $n_{r}(K)=n_{r+i}(K)=1$ for all $i=1, \ldots, r-1$.

P roof. Let $S$ be some Sylow $r$-subgroup of $K$ and $\Sigma$ the points stabilized by $S$. By Lemma 3.3, the size of $\Sigma$ is $r-1$. Let $A$ be the set of all $S$-orbits that have length $r$. If $N$ denotes the normalizer of $S$ in $K$, then $N$ permutes the sets in $A$ and so $A$ is partitioned into $N$-orbits. From each such orbit we select one $r$-set, resulting in a collection $\lambda_{1}, \ldots, \lambda_{s}$. The proposition will now be proved in 6 steps.

Cla im 1. $n_{r}(K)=s=n_{2 r-1}(K)$.

P r o of. As $S$ fixes precisely $r-1$ points, an $r$-element set is $S$-invariant if and only if the set belongs to $A$. Thus $\Lambda^{K}=\left\{\lambda^{k} \mid k \in K, \lambda \in \Lambda\right\}$ is the system of all $r$-element sets left invariant under conjugates of $S$. As, by assumption, every $r$-element set is left invariant by a Sylow $r$-subgroup, $\Lambda^{K}=\Omega^{\{r\}}$. Therefore each $K$-orbit on $\Omega^{\{r\rangle}$ contains at least one of the $\lambda_{t}$, and so by Result 2.4, exactly one of the $\lambda_{i}$. Thus $n_{r}(K)=s$. Now observe that a $(2 r-1)$-element set is invariant under $S$ if and only if it is of the form $\Sigma \cup \lambda$ for some $\lambda$ in $A$. As above we conclude that $n_{2 r-1}(K)=s$. This completes the proof of Claim 1 and Proposition 4.1 i).

From now onward we may assume that $r$ is at least 3 , the case $r=2$ is dealt with in Proposition 1 in [4]. If $4 r-2 \leqq n$, then Claim 1 and Theorem 1 in [7] implies $n_{r}(K)=n_{r+1}(K)=\cdots=n_{2 r-1}(K)$. It remains to show that $s=1$.

$\mathrm{Cla \textrm {im }} 2$. For every $k \leqq r-1$ and every $\lambda$ in $A$ the group $N_{(\lambda)}$ acts $k$-fold homogeneously on $\Sigma$.

P roof. Let $\Gamma$ be a $k$-subset of $\Sigma$ and consider the sets $\Gamma \cup \lambda_{1}, \ldots, \Gamma \cup \lambda_{s}$. By Result 2.4 these sets belong to different $K$-orbits and as there are only $s=n_{r+k}(K)$ orbits on $\Omega^{\{r+k\}}$ (by Claim 1 and the subsequent remark), each $K$-orbit contains precisely one of these sets. Suppose now that $\Delta$ is some other $k$-subset of $\Sigma$. Then some $g$ in $K$ maps $\Gamma \cup \lambda_{1}$ onto $\Delta \cup \lambda_{i}$ for some $i$. By Result 2.4 we may assume that $g$ belongs to $N$ and therefore $i=1$. Thus $g$ maps $\Gamma$ onto $\Delta$. As this may be done independently of $\lambda$, the result follows.

We shall say that $\lambda_{i}$ and any $r$-element subset in the same $K$-orbit as $\lambda_{i}$ has colour $\underline{i}$. The colour content of a set of size at least $r$ is any sequence of the colours of all its $r$-element subsets. Next we determine the colour contents of all $(r+1)$-element subsets. We choose $\alpha$ in $\Sigma$. Then, as $N_{\alpha,\left(\lambda_{i}\right)}$ is transitive on $\lambda_{i}$, the colour contents of $\alpha \cup \lambda_{i}$ is of the form $\left(\underline{c}_{i}, \underline{c}_{i}, \ldots, \underline{c}_{i}, \underline{i}\right)$ where $\underline{c}_{i}$ is some colour depending on $i$, not necessarily different from $\underline{i}$. 
C1aim 3. Every $(r+1)$ element set has colour content $\left(\underline{c}_{i}, c_{i}, \ldots, \underline{c}_{i}, \underline{i}\right)$ for some $i=1,2, \ldots, s$. In particular, if an $(r+1)$-elements set contains at least two sets of equal colour, then it contains at least $r$ sets of the same colour.

P r o of. In all we get $s=n_{r+1}(K)$ distinct colour contents of type $\left(\underline{c}_{i}, \underline{c}_{l}, \ldots, \underline{i}\right)$ for $(r+1)$-sets. As sets in the same orbit must have the same colour content, these are already all colour contents.

$\mathrm{Cla} \mathrm{im} \mathrm{4.} \mathrm{For} \mathrm{any} \mathrm{two} \mathrm{distinct} \mathrm{colours} \underline{i}$ and $j$ there is an $(r+1)$-element set containing $\underline{i}$ and $j$ simultaneously. In particular, $s \leqq 3$.

P r o of. As $K$ is $(r-1)$-fold homogeneous, for a suitable $g$ in $K, \lambda_{i}$ and $\lambda_{j}^{g}$ intersect in $r-1$ points. So the union of these sets has the desired property. By Claim 3 , an $(r+1)$ element set accomodates at most two colours simultaneously, hence we have $\left(\begin{array}{l}s \\ 2\end{array}\right) \leqq s$ and
so $s \leqq 3$.

$\mathrm{Cla}$ i $\mathrm{m}$. There is a monochromatic set of size $r+1$. In particular, $s \leqq 2$.

P r o of. Let $\alpha^{\prime}$ be some point different from $\alpha$ in $\Sigma$ and put $\lambda=\lambda_{1}$. Consider the sets $\Gamma=\{\alpha\} \cup\left\{\alpha^{\prime}\right\} \cup \lambda$ and $\Gamma_{\alpha^{\prime}}=\Gamma \backslash\left\{\alpha^{\prime}\right\}$. By definition the latter set has colour content $(\underline{c}, \underline{c}, \ldots, \underline{c}, \underline{1})$ where $\underline{c}=\underline{c}_{1}$. According to Claim 2, this is also the colour content of $\Gamma_{\alpha}=\Gamma \backslash\{\alpha\}$. Thus we have accounted for the colours of all subsets of $\Gamma$ that contain at most one of $\alpha$ and $\alpha^{\prime}$. The remaining sets are $\Gamma_{\beta}=\{\alpha\} \cup\left\{\alpha^{\prime}\right\} \cup \lambda \backslash\{\beta\}$ for some $\beta$ in $\lambda$. Such a set contains $\Gamma_{\alpha} \backslash\{\beta\}$ and $\Gamma_{\alpha^{\prime}} \backslash\{\beta\}$, both of colour $\underline{c}$. By Claim 3 , the colour content of $\Gamma_{\beta}$ is $\left(\underline{c}, \underline{c}, \ldots, \underline{c}, \underline{c}_{\beta}\right)$ where $\underline{c}_{\beta}$ depends only of the choice of $\beta$ in $\lambda$. We shall show that $\underline{c}_{\beta}=\underline{c}$ for at least some $\beta$ in $\lambda$, thus producing the monochromatic set $\Gamma_{\beta}$.

Assume to the contrary that $\underline{c}_{\beta} \neq \underline{c}$ for all $\beta$. In $\Gamma_{\beta}$ let $\Gamma_{\beta} \backslash\left\{\beta^{\prime}\right\}$ be the set of colour $\underline{c}_{\beta}$. Then this set also is the only set in $\Gamma_{\beta}$, of colour different from $\underline{c}$. In this way we obtain a pairing $\Gamma_{\beta} \leftrightarrow \Gamma_{\beta^{\prime}}$, which contradicts the fact that $|\lambda|=r$ is an odd prime. Hence $(\underline{c}, \underline{c}, \ldots, \underline{c})$ is the colour content of an $(r+1)$-element set. This implies that $s \leqq 2$.

Claim 6. $s=1$.

P r o of. For $s=2$, the only colour contents are $\underline{a}:(\underline{1}, \underline{1}, \ldots, \underline{1}, \underline{2})$ and $(\underline{2}, \underline{2}, \ldots, \underline{2}, \underline{2})$ or $\underline{b}:(1, \underline{1}, \ldots, \underline{1}, 2)$ and $(1,1, \ldots, 1,1)$. This follows from Claims $3-5$. Possibility $\underline{a}$ does not occur: according to the proof of Claim 1, we may assume that the sets $\{\alpha\} \cup \lambda_{1}$ and $\{\alpha\} \cup \lambda_{2}$ represent the two orbits on $(r+1)$-element sets. However, their colour contents are not of this form. To rule out possibility $\underline{b}$, let $\Delta=\lambda_{2} \backslash\{\beta\}$ for some $\beta$ in $\lambda_{2}$. Then $\lambda_{2}$ is the only set of colour 2 containing $\Delta$, for otherwise the colour content of some $(r+1)$-element set would be $(2,2, \ldots$,$) . Therefore any subgroup fixing \Delta$ setwise must fix the point $\beta$. Considering a Sylow $r$-subgroup of $K_{(\Delta)}$ we conclude that the Sylow subgroup fixes at least $r$ point. This contradicts Lemma 3.3. Hence $s=1$.

This completes the proof of Proposition 4.1.

5. Proof of Theorem A. Suppose first that $4 r-2 \leqq n$. By Proposition $4.1 K$, hence also $H$, is transitive on $(2 r-1)$-sets. From Theorem 2 in [7] it follows that $H$ is at least $(2 r-2)$-transitive, hence $r$ divides the order of $H$, which contradicts the assumption. 
Suppose next that $r \geqq 7$; then $H$ is $(r-1)$-transitive by Lemma 3.3 and Theorem 2 in [7]. By Lemma 10 in [7] there is a set $\Delta$ of size $r$ such that $H_{(\Delta)}^{\Delta} \supseteqq \mathbb{A}^{\Delta}$. This contradicts the fact that $r$ does not divide the order of $H$.

It remains to consider the case that $r \leqq 5$ and $4 r-2>n$. Beaumont and Peterson [1] determined all finite permutation groups $G$ on a set $\Omega$ with $G \approx \mathbb{A}^{\Omega}$ and $G \geqq \mathbb{A}^{\Omega}$. Of the four groups with this property three meet our arithmetic condition and they are the cases (iii), (vi) and (vii) in the table. Henceforth we shall assume that $K \geq \mathbb{A}^{\Omega}$.

By Lemma $3.3 K$ is $(r-1)$-transitive. Since $n \equiv r-1(r)$ the only possibilities are that $n=2 r-1$ or $n=3 r-1$. The former case is ruled out by well-known theorems of Jordan, see Wielandt [10] Theorems 13.3 and 13.9 .

Suppose $r=5$. Then $K$ is a 4-transitive group of degree 14. It follows that $K$ contains an 11-cycle, but this case is again ruled out by Theorem 13.9 in [10].

Suppose $r=3$. The only 2-transitive groups of degree 8 not containing $\mathbb{A}_{8}$ are: $A_{1}(8)$, $\Gamma A_{1}(8), 2^{3} \backslash P S L_{3}(2), P S L_{2}(7)$ and $P G L_{2}(7)$. The order of $A_{1}(8)$ is not divisible by 3 , hence this group cannot occur here. We now consider $P S L_{2}(7)$ and $P G L_{2}(7)$. Let $\Delta$ be a set of 4 harmonic points on the projective line over $F_{7}$. The cross-ratio of these points is $-1,2, \frac{1}{2}$, dependent on the order in which they are taken, and a cyclic group of order 3 applied to $\Delta$ permutes cyclically these three values. However, a linear transformation leaves the cross-ratio unchanged, hence no group of order 3 in $P S L_{2}$ (7) or $P G L_{2}(8)$ can fix $\Delta$. Consequently $K$ is neither of these two groups.

It remains to show that $\Gamma A_{1}(8)$ and $2^{3} \backslash P S L_{3}(2)$ satisfy the conditions for $K$. We have

$$
A_{1}(8) \stackrel{3}{\square} \Gamma A_{1}(8) \stackrel{8}{\complement} 2^{3} \backslash P S L_{3}(2) .
$$

There are seventy 4 -sets in $\Omega$ and these cannot be in one orbit under $2^{3} \backslash P S L_{3}(2)$ since the order of this group is not divisible by 5 . We now consider $A_{1}(8)$ acting on $\Omega$. A 4-set cannot be left fixed by an element of order 7 . It is easily seen that there exists a 4-set fixed by a group of order 4 and not 8 and that there also exists a 4-set fixed only by the identity. Hence the former lies in an orbit of length 14 and the latter in an orbit of length 56 . Hence $A_{1}(8)$ has 2 orbits on the seventy 4 -sets. It follows that $2^{3} \backslash P S L_{3}(2)$, hence also $\Gamma A_{1}(8)$, have these orbits on 4-sets. By a result in [9] mentioned in the introduction it follows that the three groups are orbit equivalent. These give cases (iv) and (v) in the table.

Finally suppose $r=2$. The only transitive groups of degree 5 not containing $\mathbb{A}_{5}$ are $C_{5}$, $D_{10}$ and $A_{1}(5)$. The number of 2-sets is even and $A_{1}(5)$ is transitive on 2-sets, hence no Sylow 2-subgroup of $A_{1}(5)$ fixes a 2-set. Now $C_{5} \subset D_{10}$ and they both have two orbits of length 5 on 2 -sets. This gives case (ii) in the table.

\section{A variant of Theorem $A$.}

Theorem B. Let $K$ be a finite primitive permutation group on a set $\Omega$ and $K \geq \mathbb{A}^{\Omega}$. Then the following conditions are equivalent:

(a) There exists an $H \subset K$ with $H \approx K$ and there is a prime $r$ dividing the order of $K$ but not the order of $H$. 
(b) There is a prime $r$ such that given any $\Delta \subset \Omega$ there exists a Sylow r-subgroup of $K$ leaving $\Delta$ fixed as a set.

(c) $K=D_{10}$ and $|\Omega|=5, K=\Gamma A_{1}(8)$ or $2^{3} \backslash P S L_{3}(2)$ and $|\Omega|=8$.

[Note: the condition that $K \geq \mathbb{A}^{\Omega}$ is essential in this theorem, since otherwise, when $|\Omega|=m r^{a}-1>9,1 \leqq m<r$, the symmetric and alternating groups satisfy (b) but $\operatorname{not}(\mathrm{a})$.]

That (a) implies (b) was noted in Section 1. That (b) implies (a) is less obvious. It follows from the considerations of Section 5 that (c) implies (a). It remains to show that (b) implies (c). The proof requires the classification of 3-transitive groups, see for example [8].

In Sections 3 and 4 only (b) was used.

Suppose that $r \geqq 7$. Then by Lemma $3.3 \mathrm{~K}$ is at least 6 -transitive, hence contains the alternating group.

Suppose $r \leqq 5$ and $4 r-2 \leqq n$. By Proposition $4.1 K$ is $(2 r-1)$-homogeneous. If $r=3$ or 5 it follows from Theorem 2 in [7] that $K$ is $(2 r-1)$-transitive. If $r=5$ then again $K$ contains the alternating group which is excluded. If $r=3$ then $K$ is 5-transitive, hence $M_{12}$ or $M_{24}$. Their degrees however are divisible by 3 , hence cannot occur in this context.

Suppose $r=2$. Then $K$ is 3-homogeneous. Suppose first that $K$ is not 3-transitive. Such groups are listed in Kantor [6] and they all have even degree, hence cannot occur in this context. Consequently we may assume that $K$ is 3 -transitive. Since the degree must be odd the only cases that need to be considered are:

(i) $P S L_{2}(q) \subseteq K \subseteq P \Gamma L_{2}(q), q=2^{a}, a>2$,

(ii) $M_{11}$ on 11 points, or

(iii) $M_{23}$ on 23 points.

Suppose (i) is the case. The number of 2-sets is even and $P S L_{2}(q)$ is transitive on 2-sets, hence no Sylow 2-subgroup fixes a 2-set.

Suppose (ii) is the case. The number of 4-sets is even and $M_{11}$ is transitive on 4-sets, hence no Sylow 2-subgroup fixes a 4-set.

Suppose (iii) is the case. We identify $\Omega$ with the points of a $4-(23,7,1)$ design on which $M_{23}$ is the automorphism group. Let $\Gamma$ consist of the points of a block together with one further point. Two 7-sets in $\Gamma$ meet in 6 points, hence $\Gamma$ contains a unique block, say $\Sigma$. A Sylow 2-subgroup of $M_{23}$ fixing $\Gamma$ must also fix $\Sigma$, hence must fix at least one point in $\Sigma$ and also the point in $\Gamma-\Sigma$. This contradicts the fact that a Sylow 5-subgroup of $K$ has only one fixed point.

There remains the case $r \leqq 5$ and $4 r-2>n$. This case was dealt with in Section 5 using only condition (b).

\section{References}

[1] R. A. Beaumont and R. P. Peterson, Set-transitive permutation groups. Canad. J. Math. 7, 35-42 (1955).

[2] R. D. Bercov and C. R. HobBy, Permutation groups on unordered sets. Math. Z. 115, 165-168 (1970).

[3] D. Betten, Geometrische Permutationsgruppen. Mitt. Math. Ges. Hamburg 10, 317-324 (1977).

[4] P. Cameron, P. M. Neumann and J. SaXL, An interchange property in finite permutation groups. Bull. London Math. Soc. 11, 161-169 (1979). 
[5] M. HaLl, The theory of groups. New York 1959.

[6] W. M. KANTOR, $k$-homogeneous groups. Math. Z. 124, 261-265 (1972).

[7] D. Livingstone and A. WAGNER, Transitivity of finite permutation groups on unordered sets. Math. Z. 90, 393-403 (1965).

[8] E. E. ShULT, Permutation groups with few fixed points. In: P. Plaumann and K. Strambach (Eds.), Geometry - von Staudt's Point of View, 275-311; Dordrecht 1981.

[9] J. Siemons, On partitions and permutation groups on unordered sets. Arch. Math. 38, 391-403 (1982).

[10] H. WIELANDT, Finite permutation groups. New York-London 1964.

Eingegangen am 22.10.1984

Anschrift der Autoren:

Johannes Siemons

Rittnertstr. 53

D-7500 Karlsruhe 41
Ascher Wagner

Department of Mathematics

University of Birmingham

Birmingham 15

England 